\title{
The Thermal Diffusivity of Glass Sieves: II. Gas-saturated Frits
}

\section{Ulf Hammerschmidt ${ }^{1}$ (D) Muhammad Abid $^{2}$}

Received: 14 March 2021 / Accepted: 1 April 2021 / Published online: 3 May 2021

(C) The Author(s) 2021

\begin{abstract}
The thermal diffusivity of gas-saturated glass sieves (frits) of porosities between $20 \%$ and $48 \%$ is presented as measured at room temperature and ambient pressure. The saturants cover a range in thermal diffusivity from $20.63 \times 10^{-6} \mathrm{~m}^{2} \cdot \mathrm{s}^{-1}$ to $172 \times 10^{-6} \mathrm{~m}^{2} \cdot \mathrm{s}^{-1}$. The experiments were carried out using a transient hot-bridge instrument of an expanded uncertainty of $5 \%$ to $10 \%$. It turned out that all measured thermal diffusivities of the gas-saturated frits (1) are smaller than that of the borosilicate glass the frits are made from and (2) are completely different from the thermal diffusivities of the liquid-saturated frits. Both these discrepancies can be attributed to characteristic properties of the matrix and not directly to the saturants. The first divergence results from thermal tortuosity that lengthens the pathway of heat through the matrix, whereas the second arises from dissolved He that enhances the transport of heat through the matrix.
\end{abstract}

Keywords Dissolved helium · Frit · Glass sieve · Gas saturant · Porous medium · Thermal conductivity $\cdot$ Thermal porosity $\cdot$ Transient hot-bridge method

\section{Introduction}

In three previous reports [1-3], the authors presented the thermal conductivity of liquid- and gas-saturated glass sieves as well as the thermal diffusivity of liquidsaturated glass sieves, so-called frits, at room temperature and atmospheric pressure. As tested at the same standard conditions, this fourth report deals with the thermal diffusivity of gas-saturated frits.

Again, the same nine frits of distinct porosities between $20 \%$ and $48 \%$ have successively been completely filled with five different gases and gas mixtures of thermal

Ulf Hammerschmidt

ulf.hammerschmidt@ outlook.com

1 Physikalisch-Technische Bundesanstalt (PTB), Bundesallee 100, 38116 Braunschweig, Germany

2 Department of Mechanical Engineering, COMSATS University Islamabad, Wah Campus,

Islamabad, Pakistan 
Table 1 Thermophysical properties of borosilicate glass 3.3 at $20{ }^{\circ} \mathrm{C}$ [4]. The volumetric specific heat, $\left(\rho c_{p}\right)$, is defined by the arithmetic product of density, $\rho$, and specific heat, $c_{p}$

\begin{tabular}{lllll}
\hline $\begin{array}{l}\text { Thermal conductivity } \\
{\left[\mathrm{W} \cdot \mathrm{m}^{-1} \cdot \mathrm{K}^{-1}\right]}\end{array}$ & $\begin{array}{l}\text { Thermal diffusivity } \\
{\left[\mathrm{m}^{2} \cdot \mathrm{s}^{-1}\right]}\end{array}$ & $\begin{array}{l}\text { Vol. Spec. Heat } \\
{\left[\mathrm{J} \cdot \mathrm{m}^{-3} \cdot \mathrm{K}^{-1}\right]}\end{array}$ & $\begin{array}{l}\text { Spec. Heat } \\
{\left[\mathrm{J} \cdot \mathrm{m}^{-3} \cdot \mathrm{K}^{-1}\right]}\end{array}$ & $\begin{array}{l}\text { Density } \\
{\left[\mathrm{kg} \cdot \mathrm{m}^{-3}\right]}\end{array}$ \\
\hline 1.138 & $0.63 \times 10^{-6}$ & $1.784 \times 10^{6}$ & $0.8 \times 10^{3}$ & $2.23 \times 10^{3}$ \\
\hline
\end{tabular}

diffusivities between $20.63 \mathrm{~mm}^{2} \cdot \mathrm{s}^{-1}$ and $172.5 \mathrm{~mm}^{2} \cdot \mathrm{s}^{-1}$ and analyzed. The runs on these gas/solid composite media were performed using a transient hot-bridge instrument of an expanded $(k=2)$ measurement uncertainty of $5 \%$ to $10 \%$. The experimental datasets could readily be evaluated with the further knowledge of some characteristic transport phenomena of the carefully studied frits already disclosed in the framework of the previously published reports [1-3].

The glass sieves under test, the gas saturants, and the transient hot-bridge instrument have already been described in detail. In like manner, the theoretical basics have been introduced and discussed. Hence, the present report comprises only brief representations of the materials and methods applied. The focus has been laid on the so-far still missing experimental results and their data reduction. For the latter task, the theoretical fundamentals have already been described in detail. Here, only the basic relevant information is sketched out.

\section{Materials and Methods}

\subsection{Glass Sieves}

The nine frits under test were provided by the manufacturer "ROBU Glasfilter GmbH," Hattert, Germany [4]. The specimens are made of borosilicate glass 3.3 (Table 1). Of each frit class, here termed "P000" to "P5," two identical plates ("specimen halves"), each of size $80 \times 50 \times 10 \mathrm{~mm}^{3}$, were obtained (Table 2). During a run, the sensor of the transient hot-bridge (THB) instrument is clamped between two halves furnishing a specimen of overall thickness $20 \mathrm{~mm}$ (Table 3).

\subsection{Saturants}

The two rare gases argon and helium as well as nitrogen were selected to saturate the frits under test. In order to fill the gap in thermal diffusivity between the numerical values of nitrogen on the one hand and helium on the other, two of their mixtures were additionally applied, i.e., $50 \mathrm{~N}_{2}-50 \mathrm{He}$ and $20 \mathrm{~N}_{2}-80 \mathrm{He}$ (xxVol \%-yyVol \%). On the whole, the saturants vary in their thermal diffusivity at room temperature and atmospheric pressure between $20.63 \times 10^{-6} \mathrm{~m}^{2} \cdot \mathrm{s}^{-1}$ and $172 \times 10^{-6} \mathrm{~m}^{2} \cdot \mathrm{s}^{-1}$. Figure 1 shows the values of the thermal diffusivities of the gases vs. their thermal conductivities [5]. 
Table 2 Pore sizes, grain sizes, porosities, and inner surfaces of classes "Pxxx" of frits [4]

\begin{tabular}{llllll}
\hline Class & Pore size $[\mu \mathrm{m}]$ & $\begin{array}{l}\text { Grain size } \\
{[\mu \mathrm{m}]}\end{array}$ & Porosity $[\%]$ & $\begin{array}{l}\text { Thermal } \\
\text { Porosity } \\
{[\%]}\end{array}$ & $\begin{array}{l}\text { Inner surface } \\
{\left[\mathrm{m}^{2} \cdot \mathrm{g}^{-1}\right]}\end{array}$ \\
\hline P000 & $500-1000$ & $<900$ & 20 & 57 & n.k \\
P00 & $250-500$ & $600-900$ & 30 & 67 & 0.015 \\
P0 & $160-250$ & $366-600$ & 33 & 70 & 0.020 \\
P1 & $100-160$ & $200-355$ & 34 & 71 & 0.085 \\
P2 & $40-100$ & $90-200$ & 36 & 73 & 0.130 \\
P23 & $40-60$ & $90-150$ & 39 & 76 & 0.220 \\
P3 & $16-40$ & $<80$ & 41 & 78 & 0.350 \\
P4 & $10-16$ & $<40$ & 42 & 79 & 0.500 \\
P5 & $1.0-1.6$ & $<10$ & 48 & 85 & 1.750 \\
\hline
\end{tabular}

The term "thermal porosity" is defined in Sect. 3.1

Table 3 Selected material properties of saturant gases and gas mixtures at room temperature and atmospheric pressure [5]

\begin{tabular}{lllc}
\hline Gas & $\begin{array}{l}\text { Thermal conductivity } \\
{\left[\mathrm{W} \cdot \mathrm{m}^{-1} \cdot \mathrm{K}^{-1}\right]}\end{array}$ & $\begin{array}{l}\text { Thermal diffusivity } \\
{\left[\mathrm{m}^{2} \cdot \mathrm{s}^{-1}\right]}\end{array}$ & $\begin{array}{l}\text { Vol. specific heat } \\
{\left[\mathrm{J} \cdot \mathrm{m}^{-3} \cdot \mathrm{K}^{-1}\right]}\end{array}$ \\
\hline Argon & 0.018 & $20.63 \times 10^{-6}$ & 872.5 \\
Nitrogen & 0.026 & $21.50 \times 10^{-6}$ & 1209.3 \\
$50 \mathrm{~N}_{2}-50 \mathrm{He}$ & 0.058 & $56.48 \times 10^{-6}$ & 1026.9 \\
$20 \mathrm{~N}_{2}-80 \mathrm{He}$ & 0.100 & $108.30 \times 10^{-6}$ & 923.4 \\
Helium & 0.150 & $172.50 \times 10^{-6}$ & 869.6 \\
\hline
\end{tabular}

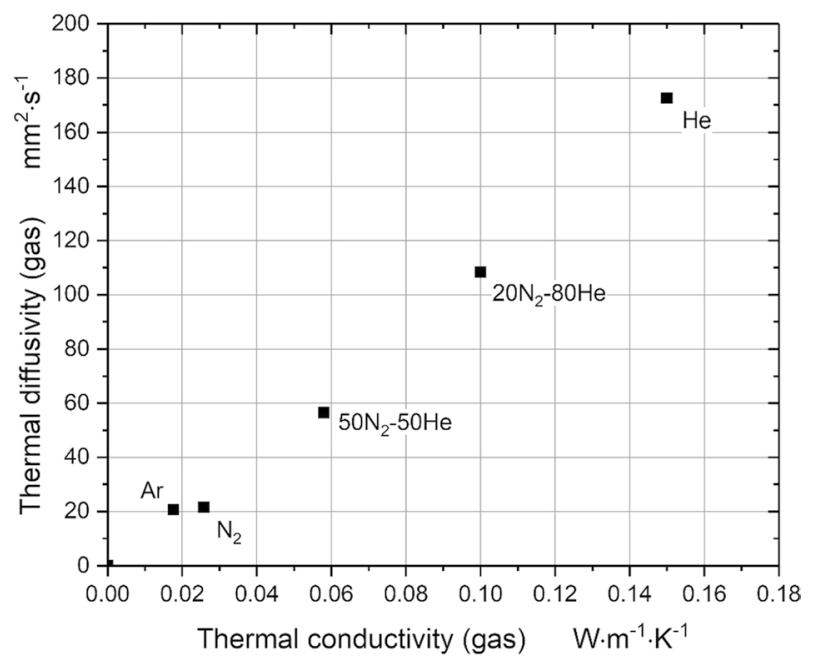

Fig. 1 Thermal diffusivity vs. thermal conductivity of saturant gases 


\subsection{Transient Hot-bridge Setup}

The transient hot-bridge instrument to simultaneously measure thermal conductivity and thermal diffusivity of solids and liquids has already been described in detail in, e.g., [6-8]. The expanded ISO uncertainties $(k=2)$ are $3 \% \leq \Delta \lambda / \lambda \leq 5 \%$ and $5 \% \leq \Delta a / a \leq 10 \%$, respectively. The latter numerical values are repeatedly validated using the borosilicate glass BK7 as secondary reference material for $\lambda$ and as in-house reference material for $a[9,10]$.

For a gas-saturated frit, a run takes less than one minute to reach the maximum probing depth of $8.8 \mathrm{~mm}$ [1-3]. The temperature rise does not exceed $1.5 \mathrm{~K}$. The short measuring time and the maximum increase in temperature prevent from any significant radiative and/or convective heat transfer inside the porous medium. A detailed analysis on the potential onset of convection $\left(t_{\text {crit }}=6.5 \mathrm{~h}\right)$ can be found in [1-3]. The stated negligible share of radiation on the total transfer of heat during a run has also been justified in the latter three reports.

The THB instrument consists of the sensor and a field-programmable system source meter "Keithley 2602." The source meter provides a voltmeter and a constant current source to operate as a line recorder. It is programmed to automatically conduct a run and to simultaneously evaluate both measurands along with their uncertainties according to working equations that are given in $[7,11]$. The data reduction algorithm, the instrument applies to evaluate the measurands, is based on the transient line-heat source solution to Fourier's second law [12].

\section{Theory}

The measurand of interest here, the thermal diffusivity, $a=\lambda /\left(\rho c_{p}\right)$, is interrelated with the thermal conductivity, $\lambda$, via the volumetric specific heat $\left(\rho c_{p}\right)$. The density and the specific heat capacity are denoted by $\rho$ and $c_{p}$, respectively. It is worth noting that, in contrast to thermal conductivity, thermal diffusivity does not depend on the amount of heat transferred. That is why, the thermal diffusivity of, e.g., evacuated porous glass of any porosity is identical to that of (bulk) glass.

In case of a simple homogeneous porous medium, the so-called corresponding thermal diffusivity is given by the porosity $(\phi)$ weighted arithmetic mean of the solid $(s)$ and fluid $(f)$ thermal conductivities and vol. specific heats, respectively:

$$
a_{0}(\phi)=\frac{(1-\phi) \lambda_{s}+\phi \lambda_{f}}{(1-\phi)\left(\rho c_{p}\right)_{s}+\phi\left(\rho c_{p}\right)_{f}} .
$$

Porosity is defined as the ratio of the void volume, $V_{V}$, and the total volume, $V_{0}$, of a porous medium.

The above steady-state relation is valid only for two parallel heat pathways that are thermally completely independent of each other. The respective quantities on the righthand side of the above equation are material constants that only depend on temperature 
and pressure. The overall thermal diffusivity, $a_{0}$, is a function of two material parameters and of a geometrical quantity and, therefore, is not a material constant.

For (transient) conduction of heat through two parallel pathways in thermal contact, the following relation for the overall thermal diffusivity is valid [3]:

$$
a_{0}=a_{s}^{\frac{(1-\phi) \lambda_{s}}{(1-\phi) \lambda_{s}+\phi \lambda_{f}}} \cdot a_{f}^{\frac{\phi \lambda_{f}}{(1-\phi) \lambda_{s}+\phi \lambda_{f}}} .
$$

For a serial arrangement of two heat pathways of individual lengths $r_{s}$ and $r_{s+f}-r_{s}$, the overall thermal diffusivity is given by [3]

$$
a_{12}=\frac{a_{s} a_{f} r_{s+f}^{2}}{a_{f} r_{s}^{2}+a_{s}\left(r_{s+f}^{2}-r_{s}^{2}\right)} .
$$

Again, in contrast to $a_{s}$ and $a_{f}$, the combined thermal transport properties, $a_{0}$ and $a_{12}$, are no material constants.

\subsection{Thermal Porosity and Thermal Tortuosity}

A frit is made by sintering milled and sieved glass fragments (grains) of arbitrary shapes but similar sizes to form the desired structural part. After completion, the grains are primarily interconnected via randomly distributed small bottleneck-like links. Microscopically, such a network does not uniformly transport heat from at least two major reasons: (1) the density of heat flow through any bottleneck of a grain significantly differs from that through the bulk and (2) heat can flow through the entire volume of a grain only if there are at least one input link and one output link that are located at precisely those two opposing positions of the grain that enable through conduction. If this is not the case, such a dead-end grain will contain "stagnant heat" during any transport of heat. This geometrical attribute gives rise to a scenario where the summed volume of through-conducting grains, $V_{C}$, differs from the volume, $V_{M}=V_{0}-V_{V}$, of all grains. Thus, the thermally active porosity, $\left(1-\phi_{\text {therm }}\right)=V_{C} / V_{0}<V_{M} / V_{0}=(1-\phi)$, differs from the (standard) porosity, $(1-\phi)$. If this is the case, Eq. 1 no longer holds.

Within the (matrix-)network, the grains are not well ordered but randomly distributed. Therefore, it can be expected that a substantial number of them will not be able to through-conduct heat. It is, thus, not surprising when observing that heat is forced to flow along a tortuous pathway of length $R$ through the matrix rather than being able to take the direct path, $r$. This phenomenon is termed thermal tortuosity and numerically described by the non-dimensional ratio $\tau_{\text {therm }}=R / r \geq 1$. According to Eqs. 2 and 3, any (overall) thermal diffusivity, measured over the distance of a tortuous way, will deviate from the standard transport property $a$. 


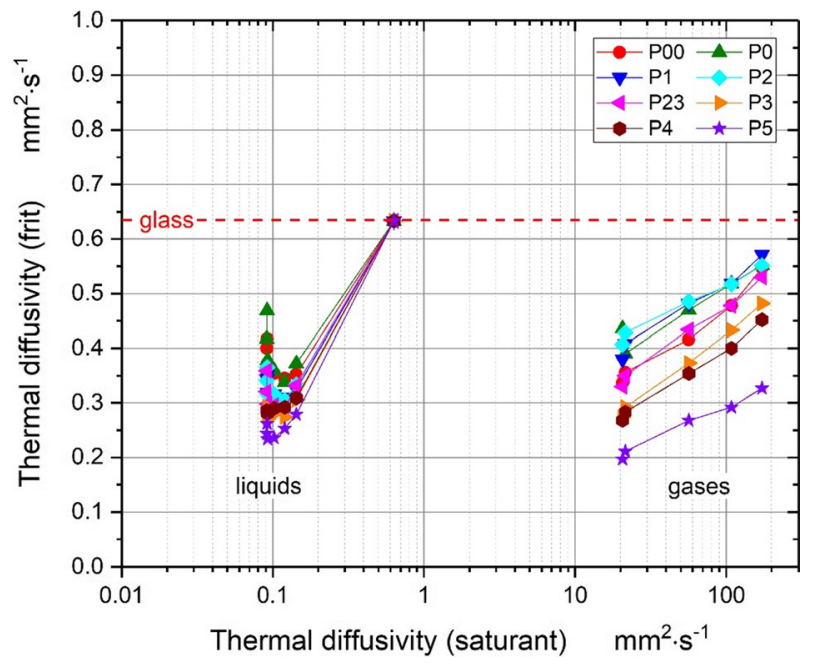

Fig. 2 Experimental thermal diffusivity of fluid saturated frits P000 to P5 vs. thermal diffusivity of saturating fluid. The horizontal logarithmic scale was chosen because it enables to compare both groups of liquid- and gas-saturated frits in one diagram

\section{Experimental Results}

Figure 2 presents the thermal diffusivities of the eight frits P000 to P5 plotted against the thermal diffusivities of their liquid and gas saturants. The log scale has only been chosen so as to compactly display the independent variable over the wide range of actual numerical values. The thermal diffusivities of the liquids vary between $0.091 \mathrm{~mm}^{2} \cdot \mathrm{s}^{-1}$ (alcohol) and $0.143 \mathrm{~mm}^{2} \cdot \mathrm{s}^{-1}$ (water), those of the gases from $20.63 \mathrm{~mm}^{2} \cdot \mathrm{s}^{-1}$ (argon) to $172.5 \mathrm{~mm}^{2} \cdot \mathrm{s}^{-1}$ (helium). At least, two key characteristics of the diagram are of special interest: (1) all measured thermal diffusivities of the LSFs and the GSFs are smaller than that of borosilicate glass $\left(a_{B G}=0.633 \mathrm{~mm}^{2} \cdot \mathrm{s}^{-1}\right)$ though even the thermal diffusivity of an evacuated frit should be equal to $a_{B G}$. Although the thermal diffusivities of all gas saturants are much larger than those of all liquid saturants, only a few of the overall thermal diffusivities of the GSFs at best are somewhat larger than those of the LSFs. This is all the more remarkable, since a linear extrapolation of the LSF datasets to larger thermal diffusivities of the saturants could be expected at overall thermal diffusivities much greater than $1 \mathrm{~mm}^{2} \cdot \mathrm{s}^{-1}$. (2) The almost linear shape of the GSF curves is significantly distinct from that of the LSF family of curves following the so-called check-mark function [3].

For comparison purposes, the thermal conductivities of the same LSFs (fitted lines) and GSFs (symbols) are given in Fig. 3. Both these families mutually are distinct as well: in contrast to the LSFs, the thermal conductivities of the GSFs do not fit the underlying $\mathrm{P}($ arallel-)S(erial-)C(onduction) model (lines) $[1,2]$. Moreover, the extrapolated fit curves of the GSF data (Fig. 2) will obviously not 


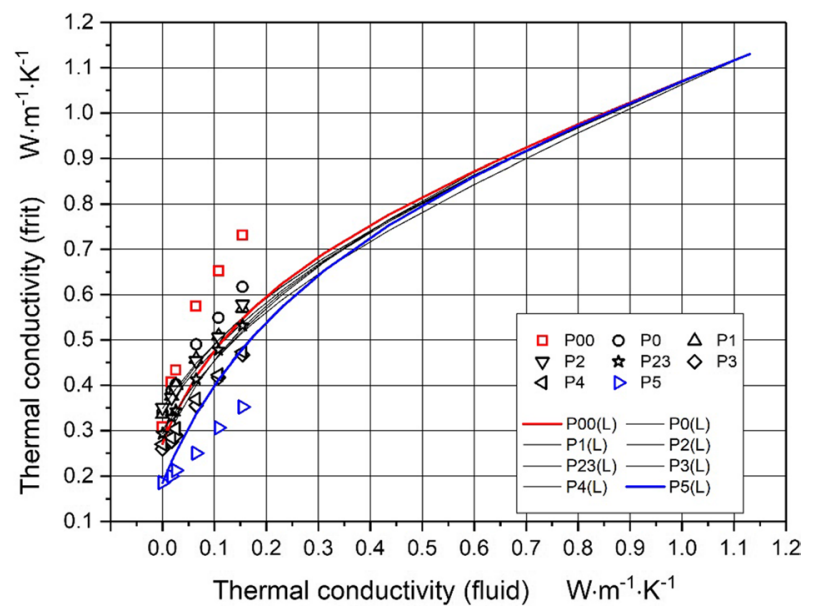

Fig. 3 Thermal conductivity of fluid saturated frits Pxx vs. thermal conductivity of saturants. Symbols indicate gas-saturated frits; lines $(\operatorname{Pxx}(\mathrm{L}))$ denote liquid-saturated frits

pass through the glass point (The fit curves are not shown in the diagram for reasons of clarity and comprehensibility).

For the above peculiarities, two major reasons have already been identified and discussed in the second report of this series [2]. Therefore, prior to the presentation of the new experimental results, it seems useful to briefly revisit some of the already published aspects of the conduction transport of heat by the frits under test [1-3].

First and foremost, the noble gas helium, used in this entire study as that saturating medium of the greatest thermal conductivity and thermal diffusivity, directly affects the thermal conductivity of each frit under test: helium diffuses into the amorphous structure of borosilicate glass where it is absorbed within the interstitial voids. At these locations, it significantly promotes the conduction transport of heat. ${ }^{1}$ Figure 4 discloses this phenomenon: the diagram shows the thermal conductivities of all frits plotted vs. the complementary thermal porosity, $\left(1-\phi_{\text {therm }}\right)$. When extrapolating the datasets of the He-containing frits to $\left(1-\phi_{\text {therm }}\right)=1$, the respective thermal conductivities of bulk borosilicate glass including dissolved $\mathrm{He}$ can be obtained. For example, borosilicate glass measured in a pure helium atmosphere might be of a $71 \%$ larger thermal conductivity than its tabulated value, $\lambda_{B G}=1.13 \mathrm{~W} \cdot \mathrm{m}^{-1} \cdot \mathrm{K}^{-1}$ (Fig. 4:"vacuum"). Even at $50 \% \mathrm{He}$ as part of the surrounding atmosphere, the thermal conductivity of the glass is increased by as much as $50 \%$ to $1.7 \mathrm{~W} \cdot \mathrm{m}^{-1} \cdot \mathrm{K}^{-1}$. By the way, any absorbed amount of He again leaves the glass when evacuating or exchanging the atmosphere to, e.g., argon or nitrogen.

\footnotetext{
1 When the experiments were planned, this effect was not considered. Unfortunately, the other gas of a particularly large thermal diffusivity, hydrogen, also diffuses into glass and, thus, is no alternative to helium as a saturant.
} 


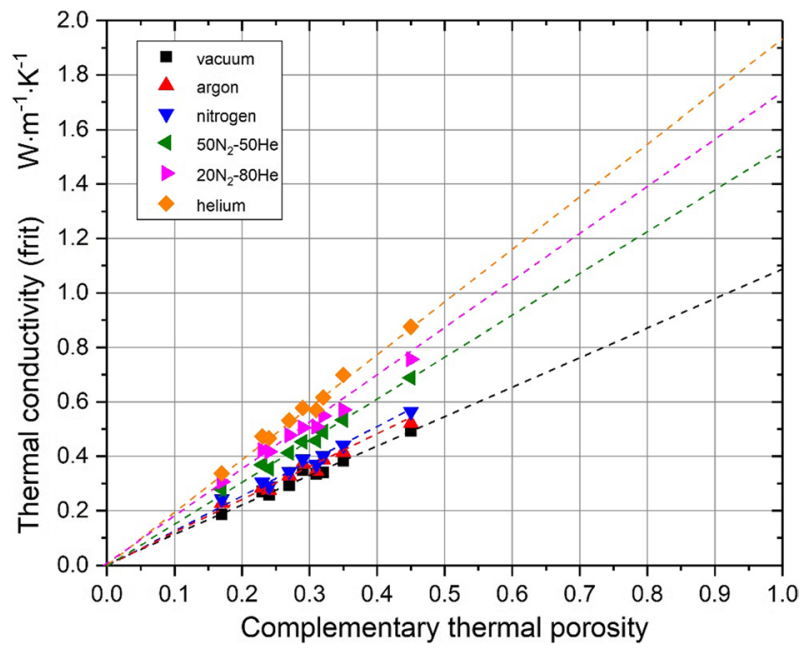

Fig. 4 Thermal conductivity of gas-saturated frits vs. complementary thermal porosity [2]

Both other gases under test, argon and nitrogen, are not able to diffuse into borosilicate glass at significant amounts $[13,14]$. Nonetheless, these latter two saturants can easily get adsorbed onto the large inner surface of each matrix (see Table 2). Here, argon and nitrogen can form multiple layers of molecules that also enhance the thermal conductivity (Fig. 4), though not as much as absorbed He.

Secondly, due to the exceedingly small thermal effusivity of all gases $\left(b \approx 6 \mathrm{~W} \cdot \mathrm{s}^{1 / 2} \cdot \mathrm{K}^{-1} \cdot \mathrm{m}^{-2}\right)$ when compared with glass $\left(b \approx 2500 \mathrm{~W} \cdot \mathrm{s}^{1 / 2} \cdot \mathrm{K}^{-1} \cdot \mathrm{m}^{-2}\right)$, the contribution of gas conduction to the overall conduction of the GSFs is relatively small. Regrettably, there are no heat transfer coefficients available in the literature for free convection between glass and gas.

The other characteristic of special interest in Fig. 2, the comparatively small thermal diffusivities of the GSFs have also been observed on the evacuated frits ("vacuum") (Figs. 5 and 6).

The following reasons were supposed to cause the above anomalies in the overall thermal diffusivities:

(1) In case of the LSFs, the t(ransient-)PSC model, Eqs. 2 and 3, correctly predicts the experimental finding: the smaller-than-expected overall thermal diffusivities of the LSFs result from a transient combination of the individual thermal diffusivities of the (solid) matrix, $a_{M}$, of the liquid within the void space, $a_{L}$, and of the combined solid and fluid medium, $a_{12}$. This means that there are three parallel paths for the conduction transport of heat, a solid, a liquid, and a mixed one, respectively. The latter path itself is of serial character.

(2) In case of the evacuated frits, it is supposed that thermal tortuosity causes the smaller-than-expected thermal diffusivities. The thermal tortuosity is determined from $\tau_{\text {therm }}=\frac{R}{r}=\sqrt{a_{B G} / a_{0}}$. As outlined in Table 4, the thermal tortuosity var- 


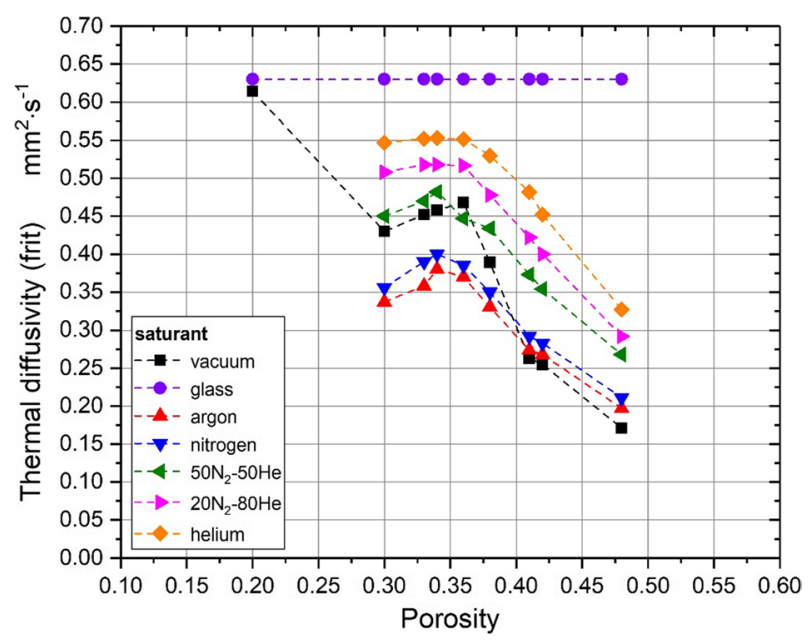

Fig. 5 Experimental thermal diffusivity of evacuated ("vacuum") and gas-saturated firts vs. porosity

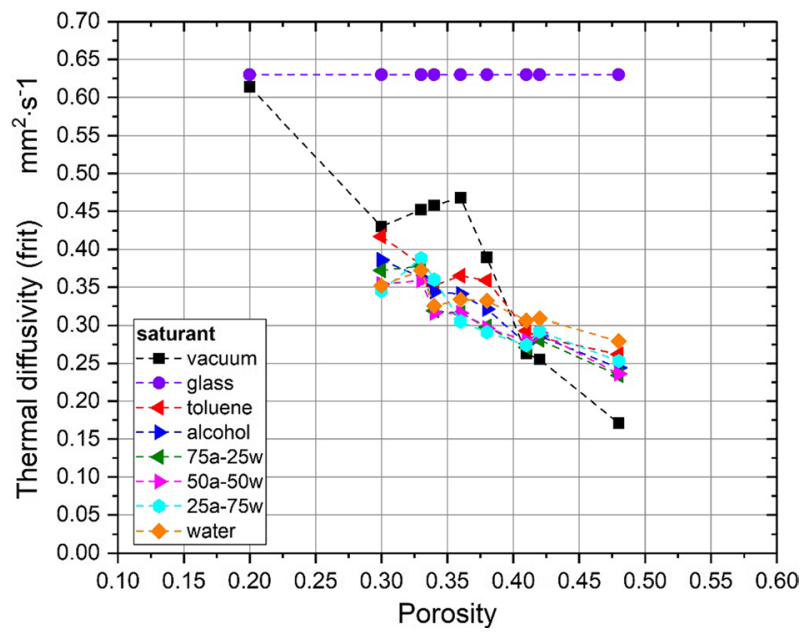

Fig. 6 Experimental thermal diffusivity of evacuated ("vacuum") and liquid-saturated frits vs. porosity

ies with the complementary porosity of the frits between 1.02 and 1.93 . While the frit P000 is of only vanishing thermal tortuosity, the heat pathlength of P5 is almost twice as that of bulk glass.

\subsection{Frit Thermal Diffusivity vs. Saturants Thermal Diffusivity}

Figure 7 presents the thermal diffusivities of all frits plotted against the thermal diffusivities of the applied gases. As shown in the diagram, the three rightmost points of each dataset, located at $a\left(50 \mathrm{~N}_{2}-50 \mathrm{He}\right)=56.48 \mathrm{~mm}^{2} \cdot \mathrm{s}^{-1}$, $a\left(20 \mathrm{~N}_{2}-80 \mathrm{He}\right)=108.3 \mathrm{~mm}^{2} \cdot \mathrm{s}^{-1}$, and $a(\mathrm{He})=172.5 \mathrm{~mm}^{2} \cdot \mathrm{s}^{-1}$, can fairly well be 
Table 4 Actual thermal diffusivity and related thermal tortuosity of borosilicate glass 3.3 and all frits under test

\begin{tabular}{llll}
\hline Specimen/matrix & $\begin{array}{l}\text { Complementary } \\
\text { thermal porosity }\end{array}$ & $\begin{array}{l}\text { Thermal } \\
\text { diffusivity } \\
{\left[\mathrm{mm}^{2} \cdot \mathrm{s}^{-1}\right]}\end{array}$ & $\begin{array}{l}\text { Thermal } \\
\text { tortuosity }\end{array}$ \\
\hline Glass & 1 & 0.633 & 1.00 \\
P000 & 0.43 & 0.614 & 1.02 \\
P00 & 0.33 & 0.430 & 1.21 \\
P0 & 0.30 & 0.452 & 1.18 \\
P1 & 0.29 & 0.458 & 1.18 \\
P2 & 0.27 & 0.468 & 1.16 \\
P23 & 0.24 & 0.389 & 1.28 \\
P3 & 0.22 & 0.263 & 1.55 \\
P4 & 0.21 & 0.255 & 1.58 \\
P5 & 0.15 & 0.171 & 1.93 \\
\hline
\end{tabular}

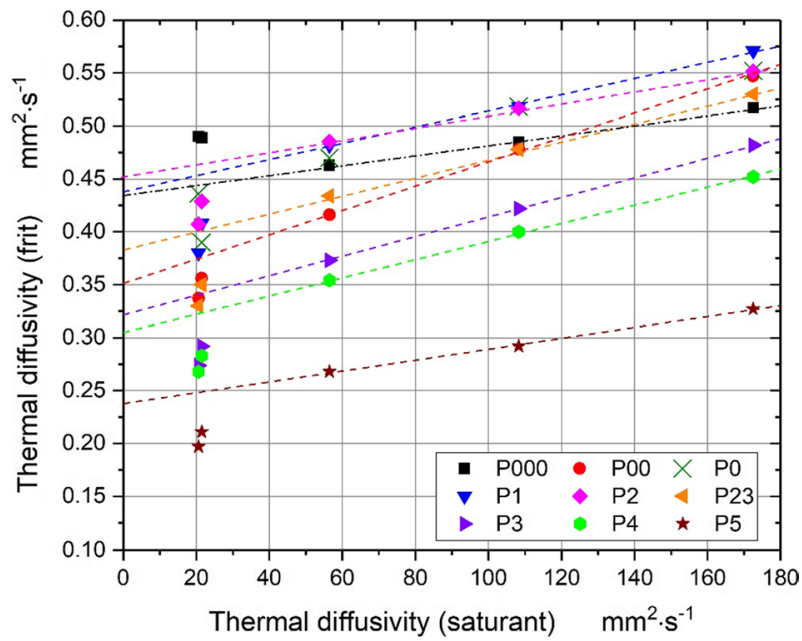

Fig. 7 Thermal diffusivity of gas-saturated frits vs. thermal diffusivity of saturant gases. The dashed lines are fits of the three rightmost data points (He-containing frits) of each set

linear fitted. The remaining two points of each set at $a(\operatorname{argon})=20.63 \mathrm{~mm}^{2} \cdot \mathrm{s}^{-1}$ and $a($ nitrogen $)=21.5 \mathrm{~mm}^{2} \cdot \mathrm{s}^{-1}$ do not match with the respective regression lines. Both latter gases yield clearly smaller overall thermal diffusivities of the frits.

The individual intercepts, $n\left(a_{S}=0\right)$, of the above eight regression lines, $a_{F}=m a_{G}+n$, are plotted vs. the respective complementary thermal porosities, $\left(1-\phi_{\text {therm }}\right)$, in Fig. 8 (black squares). The diagram demonstrates that this curve is quite similar to the one presenting the respective experimental matrix data (red circles: $a_{S}=0$ ). Therefore, it can be concluded that the above-mentioned assumption of a helium-enhanced thermal conductivity of the matrices under test likewise is applicable for the thermal diffusivity of GSFs too. Thus, frits tested inside an atmosphere of at least $50 \mathrm{Vol}$. \% He more likely behave like an evacuated 


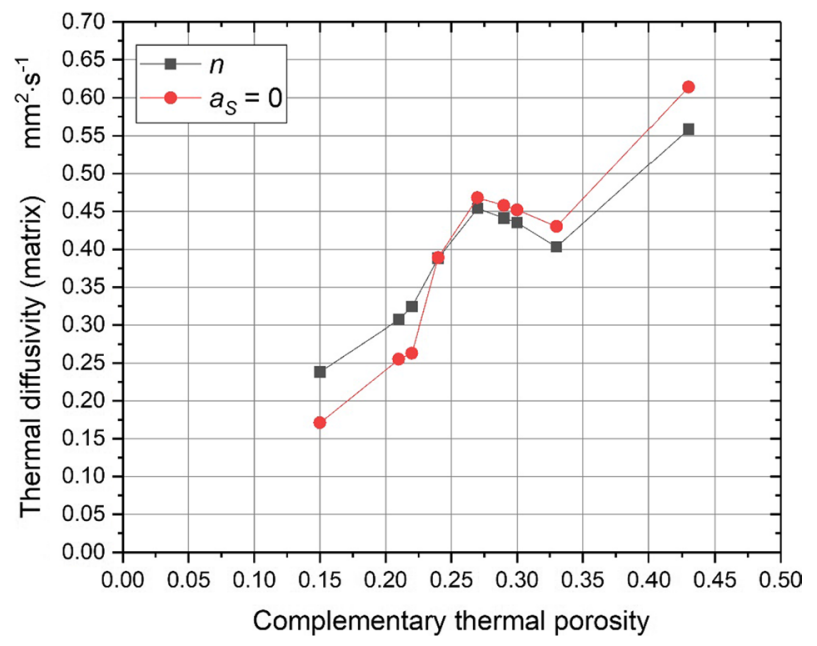

Fig. 8 Matrix thermal diffusivity vs. complementary thermal porosity (see text)

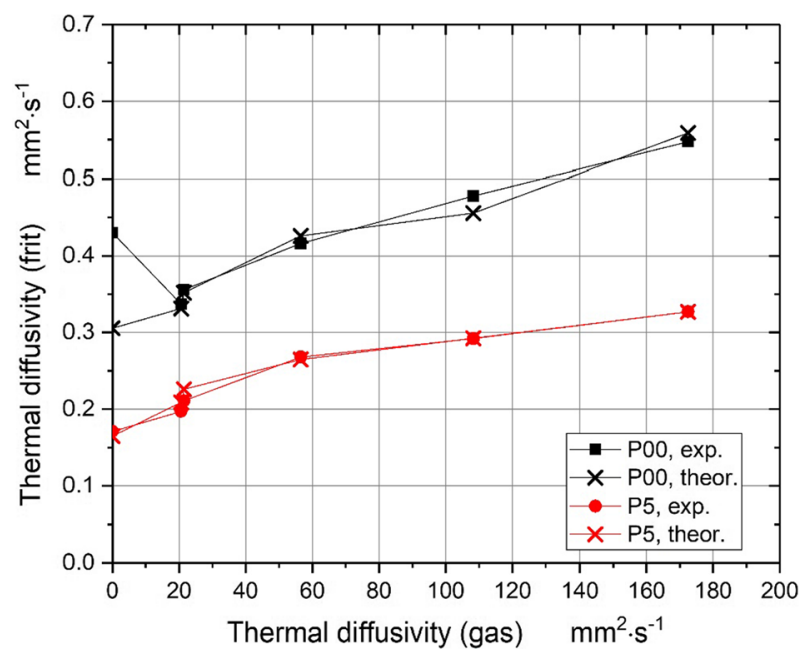

Fig. 9 Experimental and theoretical thermal diffusivities of gas-saturated frits P00 and P5 vs. thermal diffusivity of saturants along with the related matrix values

porous medium of some sort than like a liquid-saturated porous medium. The same expectation seems to be true for both $\mathrm{Ar}$ and $\mathrm{N}_{2}$ atmospheres though for distinct reason (adsorption) and varying extents.

After all, further evidence for the above empirical result is provided by the fact that all actual thermal diffusivities of the GSFs can fairly well be mathematically represented as corresponding thermal diffusivities according to Eq. 1, 


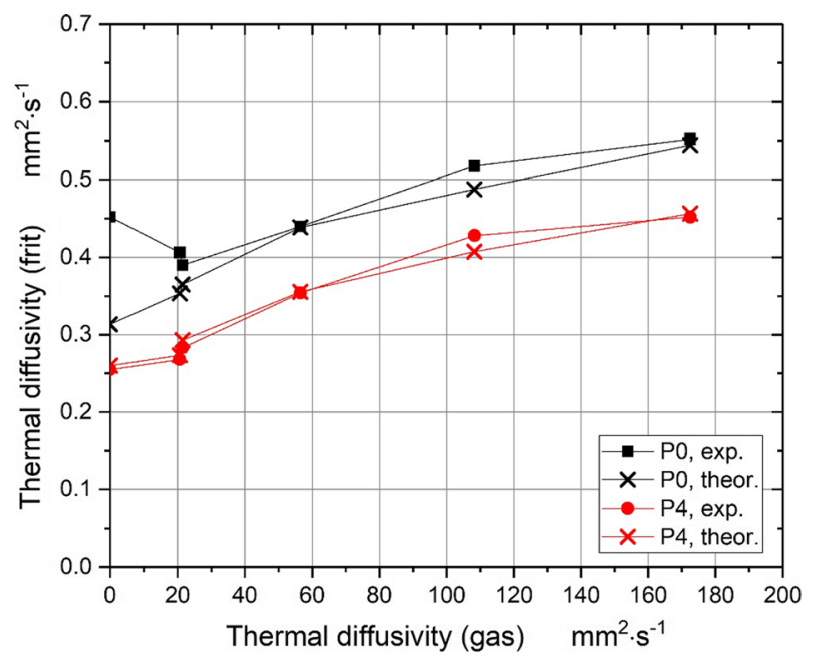

Fig. 10 Experimental and theoretical thermal diffusivities of gas-saturated frits $\mathrm{P} 0$ and $\mathrm{P} 4$ vs. thermal diffusivity of saturants along with the related matrix values

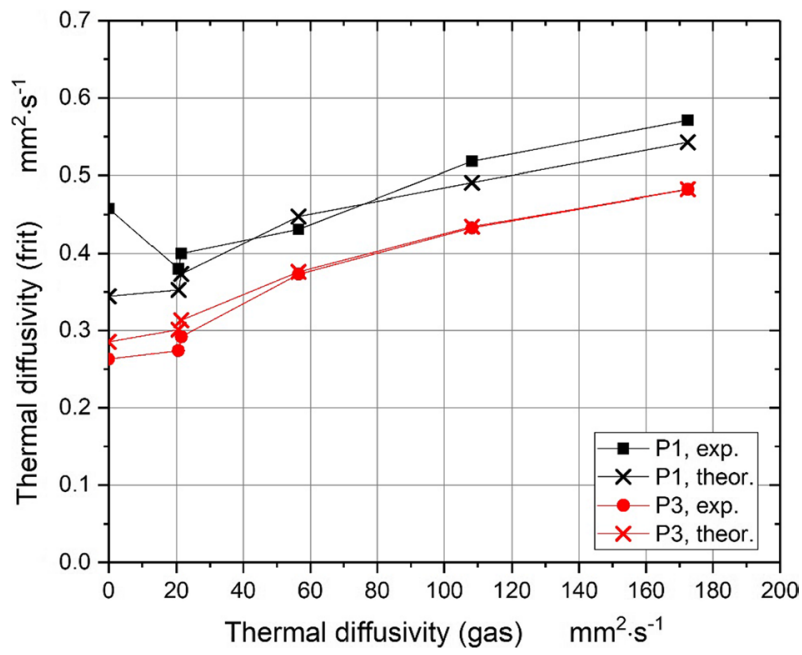

Fig. 11 Experimental and theoretical thermal diffusivities of gas-saturated frits P1 and P3 vs. thermal diffusivity of saturants along with the related matrix values

$a_{0}=\lambda_{0} /\left(\rho c_{p}\right)_{0}$ (Figs. 9, 10, 11, 12: “theor.”). However, this latter finding unconditionally requires some additional interpretation.

To calculate the corresponding thermal diffusivities of the GSFs, Eq. 1, 




Fig. 12 Experimental and theoretical thermal diffusivities of gas-saturated frits P2 and P23 vs. thermal diffusivity of saturants along with the related matrix values

$$
a_{0}=\frac{\lambda_{0}}{\left(\rho c_{p}\right)_{0}}=\frac{(1-\phi) \lambda_{M}+\phi \lambda_{S}}{(1-\phi)\left(\rho c_{p}\right)_{B G}+\phi\left(\rho c_{p}\right)_{S}},
$$

first has to be adapted to account for (1) the vanishing thermal conduction of the gas inside the voids, $\phi \lambda_{S} \rightarrow 0$, and for (2), the existence of non-through-conducting dead-end grains, $(1-\phi) \rightarrow\left(1-\phi_{\text {therm }}\right)$. Therefore, the numerator of the above equation in this case instead reads $\lambda_{0}=\left(1-\phi_{\text {therm }}\right) \lambda_{M}$. The latter dataset has already been shown in Fig. 3. Second, since $\phi\left(\rho c_{p}\right)_{S}<<(1-\phi)\left(\rho c_{p}\right)_{M}$, one finally gets for the right-hand side of Eq. 4.

$$
\frac{\left(1-\phi_{\text {therm }}\right) \lambda_{M}}{(1-\phi)\left(\rho c_{p}\right)_{B G}}
$$

Although this fraction may not be equated to $a_{0}$, it still provides reasonable numerical values for this thermal transport property as can be seen in Figs. 9, 10, 11,12 . Here, the experimental and theoretical values are contrasted to each other. The solution to this dilemma comes out of Fig. 13. This diagram presents the volumetric specific heat capacities of the evacuated and the gas-saturated frits vs. the porosities of the frits. The latter material properties were directly determined from the corresponding experimental values, $\left(\rho c_{p}\right)_{F}=\lambda_{F} / a_{F}$. Additionally, the (dashed line) standard curve according to Eq. 1 is given. The experimental matrix data deviate from that straight line because of the conduction-restricting transport phenomenon of the dead-end grains inside the matrix. Due to the conduction-enhancing He-content or $\mathrm{Ar} / \mathrm{N}_{2}$-adsorption of the matrices, the latter effect is partly compensated. The experimental curves approach the standard line. That is why, the above term, Eq. 5, can fairly well (but in principle not properly) 


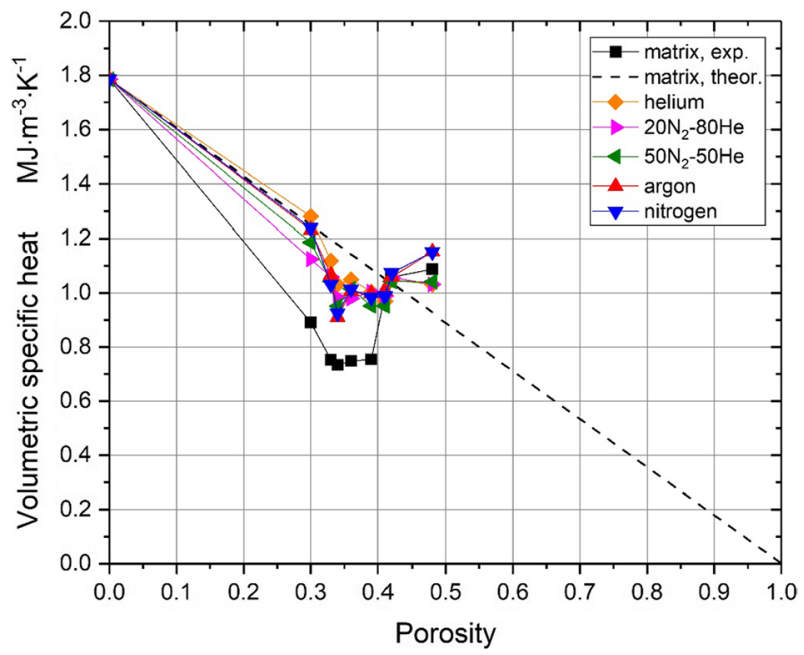

Fig. 13 Volumetric specific heat capacity of evacuated (matrix) and gas-saturated frits vs. porosity

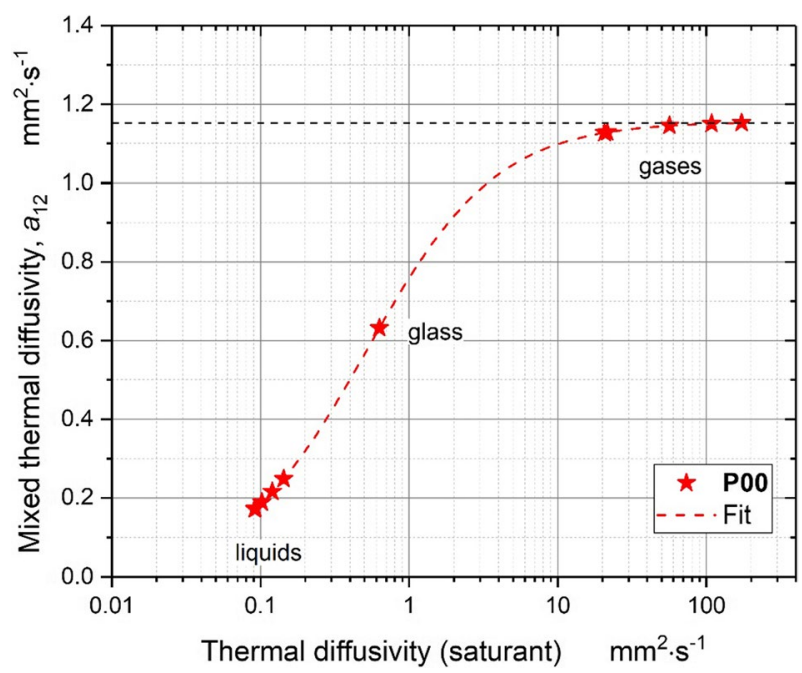

Fig. 14 Mixed thermal diffusivity of frit P00 vs. thermal diffusivity of liquid and gas saturants (cf. Fig. 2). (The log plot has only been applied for reason of clarity, nonlinear fit according to TPSC model, Eqs. 2 and 3

represent the numerical values of the overall thermal diffusivities of gas-saturated frits.

The TPSC model, Eqs. 2 and 3, with $a_{1}=a_{B G}, a_{2}=a_{G}, a_{12}=a_{12}\left(a_{B G}, a_{G}\right)$ (cf. Fig. 14) and the experimental thermal conductivities ("symbols") as shown in Fig. 3 also predict for each one of the thermal diffusivities of the GSF an almost linear dependence on the thermal diffusivity of the saturants. However, 


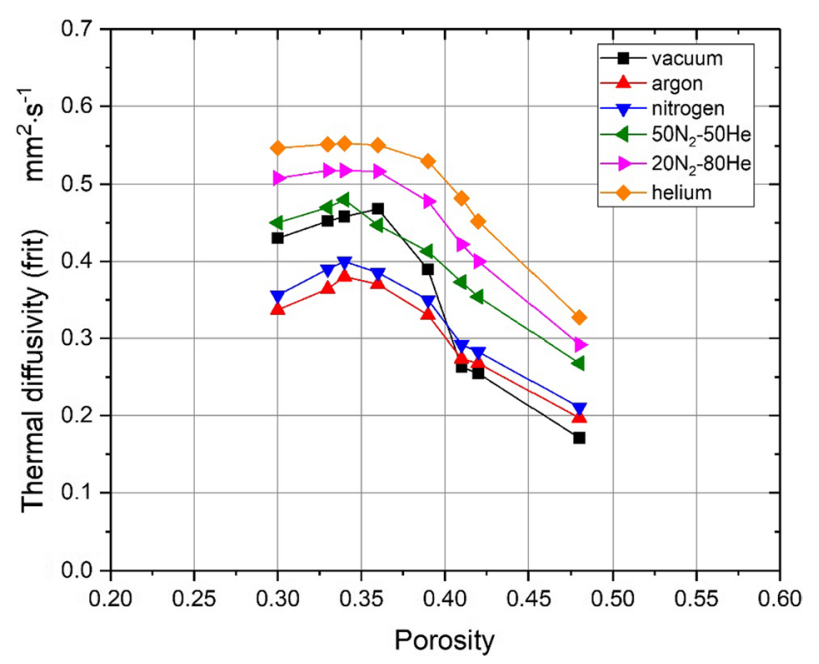

Fig. 15 Thermal diffusivities of evacuated ("vacuum") and gas-saturated frits vs. porosity. The dashed lines indicate the presumed or linear-extrapolated courses of the curves on both of their sides

the calculated numerical values are appreciably larger than the experimental ones (Figs. 9, 10, 11, 12). This latter (analytical) attribute of the TPSC model predicts the (actually vanishing) contribution of the gas inside the void space to the overall conduction of heat. Figure 14 clearly demonstrates that, in case, the gaseous saturants behave similar to the liquid saturants, and the mixed thermal diffusivities would be larger than those of bulk glass.

\subsection{Thermal Diffusivity of GSFs vs. Porosity}

The thermal diffusivities of the GSFs as functions of porosity can be compared with those of the LSFs in Figs. 5 and 6. For this purpose, the dataset of the evacuated frits (vacuum) can be used as a standard of comparison.

As is already known, the He-containing frits exhibit the larger thermal diffusivities than the $\mathrm{Ar} / \mathrm{N}_{2}$ and the evacuated ones. Figure 15 once more presents the GSF data, now, in a higher resolution. The curves roughly resemble that one of the evacuated frits ("vacuum"). All curves show a flat maximum at about $\phi \approx 0.34$.

In order to get some insight into the effects of the saturating gases on the overall thermal diffusivities, for the diagram, Fig. 16, the matrix data were treated as offset and subsequently subtracted from the data sets of the GSFs. Again, the effect of helium on the transport of heat through the borosilicate glass can clearly be demonstrated. Together with the reductions caused by the other two pure gases, Ar and $\mathrm{N}_{2}$, it is assumed that thermal tortuosity might also be individually influenced (through mixed conduction) by the applied gases. Therefore, the above data sets were recalculated in terms of tortuosity. 


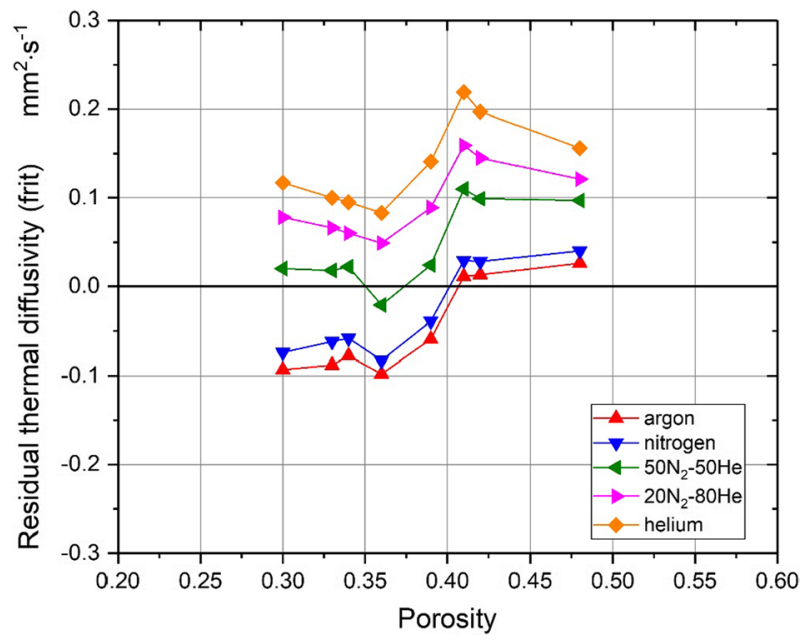

Fig. 16 Thermal diffusivity datasets as shown in Fig. 15 but now pointwise reduced by the vacuum thermal diffusivities vs. porosity

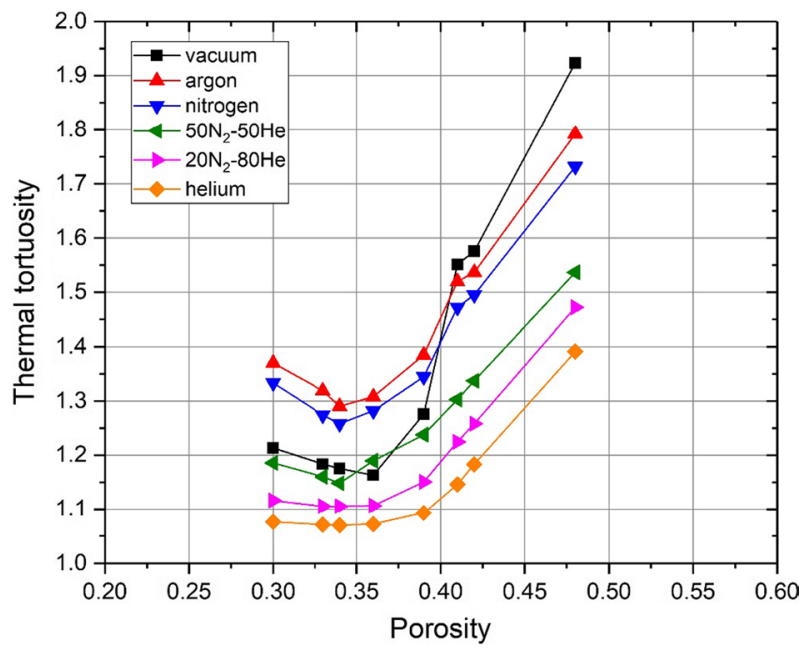

Fig. 17 Thermal tortuosity of evacuated ("vacuum") and gas-saturated frits vs. porosity

\subsection{Thermal Tortuosity}

The thermal tortuosities of the evacuated frits and the GSFs depend on porosity in a way to be seen in Fig. 17. Here, again, the virtual offset ("vacuum") has been subtracted from the other (GSFs) curves to demonstrate the effect of the applied gases on the (overall) thermal tortuosity of gas-saturated frits (Fig. 18). Surprisingly, there is an enhancement as well as a reduction in this non-dimensional parameter. Regrettably, without the further knowledge of detailed specific 


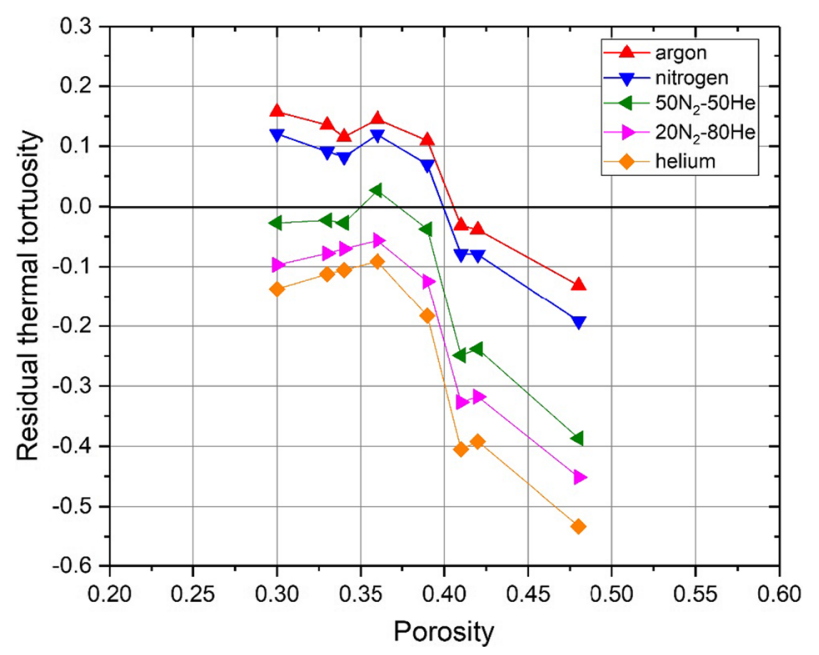

Fig. 18 Thermal tortuosity datasets as shown in Fig. 17 but now pointwise reduced by the matrix thermal tortuosity vs. porosity

morphological data of the frits, no physically confirmed statements can be made for now.

\section{Conclusion}

This last report of a four-part series on liquid- and gas-saturated borosilicate glass frits was aimed to round up the already presented image on the thermal conductivity and thermal diffusivity of these basic porous media: the thermal diffusivity of gassaturated frits was analyzed and discussed in some detail.

At the outset of the experimental, theoretical, and numerical investigations on glass sieves, the general objective was to gain a deeper insight into the transport of heat within evacuated and fluid saturated porous media. As the most promising test media, borosilicate-glass sieves had been chosen. Glass is chemically inert, and its property values are sufficiently stable, homogeneous, and well established. The frits offer a comparably broad variety in porosities at a narrow pore size distribution each. Due to their interconnected open pores, they are particularly easy to fill, to empty, to evacuate, and to clean. Specimens are available in ample supply, at minimal cost and require little or no preparation prior to a run. Especially, the relevant conduction transport properties are in a range that is readily accessible for fast and simple measurements.

The $\mathrm{T}$ (ransient)H(ot)B(ridge) method has demonstrated its ability to furnish precise measurement results on thermal conductivity and thermal diffusivity of solids and liquids. The expanded $(k=2)$ uncertainties of both measurands are estimated according to the ISO GUM and verified using the BK7 standard reference. In [1], it 
is demonstrated that neither radiative nor convective heat transfers can perturb a test run.

The experimental datasets on the thermal conductivity of evacuated and liquidsaturated frits can accurately be fitted to a simple $\mathrm{P}($ arallel)S(erial)Conduction) model. Among eleven distinct prediction models, tested for suitability, the PSC model performed best by far with maximum deviations between $-2 \%$ and $+0.5 \%$ [1]. Additionally, it helped to disclose that only the smaller volume part of each matrix is able to through-conduct heat. The larger part can contribute to the transport of heat only if the frit is saturated with a liquid. When saturated instead with a gas, the effect of mixed conduction vanishes because of an exceedingly small heat transfer coefficient [2]. In the framework of the runs on the gas-saturated frits, it was found that argon and nitrogen were adsorbed by the larger inner surfaces of the matrices whereas helium is absorbed within the glass. In both these cases, the inherent thermal conductivity of each frit is increased. The latter effect in conjunction with the minimal heat transfer from a matrix to the gas can be attributed for the origin of the so-called "thermal conductivity effect" first observed by Woodside and Messmer in 1961 and, since that time, probably misinterpreted [15, 16].

The experimental datasets on the "dynamic property" thermal diffusivity of evacuated and liquid-saturated frits cannot be described by the PSC model not even by the relation of a corresponding thermal diffusivity because of the steady-state character of these models. Since there was no transient prediction model at hand, the PSC model was extended to additionally deal with transient scenarios of "composite" solid-fluid conduction of heat. The TPSC model is able to furnish overall thermal diffusivities of serial and parallel heat flow through an arbitrary number of thermal conductors.

Funding Open Access funding enabled and organized by Projekt DEAL.

Open Access This article is licensed under a Creative Commons Attribution 4.0 International License, which permits use, sharing, adaptation, distribution and reproduction in any medium or format, as long as you give appropriate credit to the original author(s) and the source, provide a link to the Creative Commons licence, and indicate if changes were made. The images or other third party material in this article are included in the article's Creative Commons licence, unless indicated otherwise in a credit line to the material. If material is not included in the article's Creative Commons licence and your intended use is not permitted by statutory regulation or exceeds the permitted use, you will need to obtain permission directly from the copyright holder. To view a copy of this licence, visit http://creativecommons.org/licen ses/by/4.0/.

\section{References}

1. U. Hammerschmidt, M. Abid, Int. J. Therm. Sci. 96, 119 (2015)

2. U. Hammerschmidt, M. Abid, Int. J. Thermophys. 42, 40 (2021)

3. U. Hammerschmidt, M. Abid, Int. J. Thermophys. 42, 54 (2021)

4. ROBU Datenblatt und Eigenschaften VitraPOR Sinterfilter (ROBU Glasfilter-Geräte GmbH, Hattert, Germany). https://www.robuglas.com/service/technische-daten.html.

5. TUV NEL. Physical properties data services (PPDS), Thermodynamic properties database and calculation suite. https://www.tuvsud.com/en-gb/industries/chemical-and-process/flow-measurement/ consultancy-services/ppds.

6. U. Hammerschmidt, Int. J. Thermophys. 16, 557 (1995) 
7. U. Hammerschmidt, V. Meier, Int. J. Thermophys. 27, 840 (2006)

8. R. Model, R. Stosch, U. Hammerschmidt, Int. J. Thermophys. 28, 1447 (2007)

9. S. Rudtsch, R. Stosch, U. Hammerschmidt, In 16th European Conference on Thermophysical Properties, ed. by W.A. Wakeham (London, 2002)

10. S. Rudtsch, U. Hammerschmidt, Int. J. Thermophys. 25, 1475 (2004)

11. ISO/IEC GUIDE 98-3:2008, Uncertainty of measurement - Part 3: Guide to the expression of uncertainty in measurement (2008)

12. H.S. Carslaw, J.C. Jaeger, Conduction of Heat in Solids, 2nd edn. (Clarendon Press, Oxford, 1950).

13. F.J. Norton, J. Am. Ceram. Soc. 36, 90-96 (1953)

14. A. Dellis, V.K. Shah, E.A. Donley, S. Knappe, Optics Lett. 41, 2775 (2016)

15. W. Woodside, J.H. Messmer, J. Appl. Phys. 32, 1688 (1961)

16. W. Woodside, J.H. Messmer, J. Appl. Phys. 32, 1699 (1961) 